\title{
Association between the sinus microbiota with eosinophilic inflammation and prognosis in chronic rhinosinusitis with nasal polyps
}

\author{
Ji Heui Kim (1)', Sung Hee Kim², Ji Youn Lim¹, Doyeon Kim, In Seong Jeong ${ }^{1}$, Dong Kyu Lee ${ }^{1}$ and Yong Ju Jang
}

\begin{abstract}
Dysbiosis of the sinus microbiome affects the pathophysiology of chronic rhinosinusitis with nasal polyps (CRSwNPs). We investigated whether the sinus microbiota in CRSwNPs is associated with eosinophilic inflammation, especially in relation to innate lymphoid cells (ILCs), prognosis, and serum extracellular vesicles (EVs). Middle meatal swabs and serum from 31 CRSwNPs patients and six healthy controls were analyzed by 165 ribosomal RNA sequencing. ILC2s and cytokines from sinonasal tissues were measured by flow cytometry and ELISA, respectively. The relative abundances (RAs) of bacteria were compared based on eosinophilic inflammation and surgical outcome. The correlations between sinus bacteria and ILC2s, cytokines, and serum EVs were analyzed. The compositions of sinus bacteria were different between groups at the genus level. In eosinophilic CRSwNPs patients, the RA of Anaerococcus was significantly decreased $(P=0.010)$, whereas that of Lachnoclostridium was significantly increased $(P=0.038)$ compared with that in controls. The RA of Lachnoclostridium showed a significant positive correlation with interleukin (IL)-5-producing ILC2 populations ( $R=0.340, P=0.049$ ), whereas the RA of Anaerococcus showed a negative correlation with IL-5-producing ILC2 populations $(R=-0.332, P=0.055)$. The RAs of Corynebacterium, Anaerococcus, and Tepidimonas were significantly decreased in patients with suboptimal outcomes compared with those in patients with optimal outcomes and control subjects. Some sinus bacteria and serum EVs showed positive correlations. CRSwNPs patients showed distinct microbiota compositions based on eosinophilic inflammation in relation to ILC2s and surgical outcome. These findings support a relationship between the microbiota and the host immune response in CRSWNPS.
\end{abstract}

\section{Introduction}

Chronic rhinosinusitis (CRS) is a multifactorial chronic upper airway inflammatory disease. Putative pathological factors include changes in the microbiota, an imbalance of the local or systemic immune system, allergens, toxins, and genetic predisposition ${ }^{1-3}$. Several studies have reported that the microbiota may affect the pathophysiology of CRS. In general, a decrease in microbial diversity and an increase in the total number of microorganisms have been reported in patients with $\mathrm{CRS}^{4,5}$.

\footnotetext{
Correspondence: Ji Heui Kim (jhkim0217@amc.seoul.kr)

${ }^{1}$ Department of Otorhinolaryngology-Head and Neck Surgery, Asan Medical Center, University of Ulsan College of Medicine, Seoul, Republic of Korea 2Department of Otorhinolaryngology-Head and Neck Surgery, National Medical Center, Seoul, Republic of Korea
}

Therefore, dysbiosis of the microbiota could be the driving force of CRS.

Immunologic features associated with the nature or composition of the sinus microbiota could partially explain the heterogeneity of CRS. CRS with nasal polyps (CRSwNPs) is generally classified as eosinophilic or noneosinophilic, which show distinct immunological features. Eosinophilic polyps are associated with the type 2 immune response, while noneosinophilic polyps are associated with type 1 and 17 immune responses ${ }^{6}$. When a CRS patient cluster was divided into distinct subgroups according to the specific pattern of bacterial cocolonization, each subgroup was associated with a host immune response. Some subgroups exhibited increased expression of the interleukin 6, tumor necrosis factor, IL8, and IL10 
genes, whereas other subgroups were associated with induced IL5 gene expression ${ }^{7}$. Innate lymphoid cells (ILCs) are a recently identified innate lymphocyte population. Group 2 ILCs (ILC2s) secrete type 2 cytokines, such as IL-5 and IL-13, and contribute to the pathogenesis of CRSwNPs, particularly in eosinophilic tissue inflammation $^{8}$. ILCs interact with bacteria and coordinate host-bacteria relationships that are associated with the pathogenesis and progression of numerous chronic human infectious, inflammatory, and metabolic diseases ${ }^{9}$. We speculated that in CRSwNPs, ILCs could interact with the microbiota and influence eosinophil inflammation.

Bacteria secrete extracellular vesicles (EVs), including transmembrane proteins, cytosolic proteins, lipids, and nucleic acids. EVs play a role in immune and airway epithelial cell communication and can induce immune responses in allergic diseases. EVs can be detected in the blood circulation far from the local disease site ${ }^{10-13}$. In nasal lavage fluid from CRS patients, the composition of EVs secreted from microbiota has been positively correlated with bacterial composition ${ }^{14}$. We hypothesized that paranasal sinus bacteria-derived EVs can be detected in the blood. However, the relationship between sinus bacteria and serum EVs is unknown.

In the present study, the sinus microbiota in specimens from middle meatus swabs from CRSwNPs patients was analyzed based on eosinophilic vs. noneosinophilic inflammation. In addition, correlations were analyzed between sinus bacteria and ILCs and cytokines from tissues and between sinus bacteria and serum EVs.

\section{Materials and methods \\ Study populations}

This study was conducted in accordance with the Declaration of Helsinki and approved by the Institutional Review Board of Asan Medical Center (2018-0121). All participants provided written informed consent.

Thirty-one adult CRSwNP patients, including 21 eosinophilic CRSwNPs patients and 10 noneosinophilic CRSwNPs patients, and 6 healthy controls were recruited from the Department of Otorhinolaryngology-Head and Neck Surgery, Asan Medical Center, between February 2018 and December 2018. CRSwNPs was diagnosed based on the established guideline criteria of the European Position Paper on Rhinosinusitis and Nasal Polyps (2012), considering the history, nasal endoscopy findings, and computed tomography (CT) images of the paranasal sinuses $^{2}$. Eosinophilic and noneosinophilic CRSwNPs were defined based on the proportion of eosinophils $>10 \%$ and $<10 \%$, respectively, of the total inflammatory cells in the NP tissue $^{15,16}$. Subjects with antrochoanal polyps, fungal sinusitis, allergic fungal sinusitis, aspirin-exacerbated respiratory disease, immunodeficiency, pregnancy, cystic fibrosis, or primary ciliary dyskinesia were not included. All patients refrained from the use of decongestants, antibiotics, and systemic corticosteroids for at least 4 weeks prior to surgery, did not have a history of acute respiratory infection for 4 weeks before surgery, and did not have medical comorbidities that included diabetes mellitus, hypertension, or hyperlipidemia. Demographic and clinical variables recorded for each subject were age, sex, ethnicity, smoking history, allergy, asthma, antibiotic use in the past 6 months, saline rinse use, and topical steroid use in the preceding 4 weeks.

Atopic status was evaluated using a skin prick test (Allergopharma; Hermann-Körner-Straße, Reinbeck, Germany), and specific IgE antibodies against various common inhalant allergens were detected with ImmunoCAP ${ }^{\mathrm{TM}}$ tests (Thermo-Fisher Scientific, Waltham, MA, USA). Asthma and aspirin intolerance were diagnosed based on medical history. Preoperative Lund-Kennedy endoscopy score, polyp grading according to the Davos classification, Lund-Mackay CT scores, and sinonasal outcome test (SNOT-22) scores were evaluated ${ }^{2}$. Control subjects $>18$ years of age underwent septoplasty for nasal obstruction without any other inflammatory nasal diseases, such as allergic rhinitis or sinusitis, evident on CT scan.

After at least 6 months, CRSwNP patients were examined to determine changes in Lund-Kennedy endoscopy score, the need for revision of sinus procedure(s), and the use of additional courses of antibiotics or systemic steroids after routine standard perioperative administration. The optimal outcome was defined as a reduction in endoscopy scores $>50 \%$, no requirement for revision of surgical treatment, and no further use of antibiotics and systemic steroids before the 6-month follow-up visit ${ }^{17}$.

Swab samples (Isohelix DNA swab, Cell Projects Ltd., Maidstone, Kent, UK) for DNA extraction were obtained using an endoscopic guide from the middle meatus and rotated at least five full turns until saturated. The samples were placed on ice upon collection and frozen within an hour at $-80^{\circ} \mathrm{C}$ until DNA extraction. Care was taken to avoid contamination from the anterior nasal cavity during swabbing. NP tissues from CRSwNP patients and middle turbinate tissues from controls were harvested after swabbing. Blood samples were collected from the median cubital vein after overnight fasting and were transferred into serum separation tubes (BD, Franklin Lakes, NJ, USA). Serum was separated from the blood and frozen at $-80^{\circ} \mathrm{C}$ until DNA extraction.

\section{DNA extraction from samples}

Human swab samples were vortexed after dilution in $800 \mu \mathrm{l}$ of phosphate-buffered saline (PBS, Welgene, Gyeongsan, South Korea). After centrifugation at $10,000 \times g$ for $1 \mathrm{~min}$ at $4{ }^{\circ} \mathrm{C}$, the pellets of swab samples containing bacterial cells were heated for $40 \mathrm{~min}$ at $100^{\circ} \mathrm{C}$ to extract DNA from the bacterial cells. To eliminate 
remaining floating particles and debris, the supernatants were collected after centrifugation at $13,000 \mathrm{rpm}$ for $30 \mathrm{~min}$ at $4{ }^{\circ} \mathrm{C}$. For serum samples, floating particles were precipitated by centrifugation at $10,000 \times g$ for $1 \mathrm{~min}$ at $4{ }^{\circ} \mathrm{C}$, after which the supernatants were mixed with PBS. After centrifugation, bacteria and foreign particles were thoroughly eliminated by sterilizing the supernatants through a $0.22-\mu \mathrm{m}$ filter. Next, the supernatants were boiled for $40 \mathrm{~min}$ at $100^{\circ} \mathrm{C}$ to extract DNA from EVs and centrifuged at $13,000 \mathrm{rpm}$ for $30 \mathrm{~min}$ at $4{ }^{\circ} \mathrm{C}$. The supernatants were collected ${ }^{18,19}$. After this preprocessing, DNA from sinus bacteria and serum bacterial EVs was extracted using a MO BIO PowerSoil DNA Isolation Kit (QIAGEN, Hilden, Germany) and quantified using the QIAxpert system (QIAGEN).

\section{Bacterial metagenomic analysis using DNA from human samples}

Bacterial genomic DNA was amplified with the 16S_V3_F (5'-TCGTCGGCAGCGTCAGATGTGTATAAGAGACA GCCTACGGGNGGCWGCAG-3') and 16S_V4_R (5'-GT CTCGTGGGCTCGGAGATGTGTATAAGAGACAGGA CTACHVGGGTATCTAATCC-3') primers specific for the V3-V4 hypervariable regions of the $16 \mathrm{~S}$ ribosomal RNA gene. The libraries were prepared using PCR products according to the MiSeq system guide (Illumina, San Diego, CA, USA) and quantified using the QIAxpert system (QIAGEN). Each amplicon was then quantified, the equimolar ratio was obtained, amplicons were pooled, and they were sequenced on a MiSeq device (Illumina) according to the manufacturer's recommendations.

\section{Analysis of the bacterial microbiota composition}

Paired-end reads that matched the adapter sequences were trimmed using cutadapt version 1.1.6 $6^{20}$. The resulting FASTQ files containing paired-end reads were merged with The Context-Aware Scheme for Paired-End Read tool, and they were quality filtered using the Phred (Q) score based on previously described criteria ${ }^{21,22}$. Reads $<350$ and $>550 \mathrm{bp}$ after merging were discarded. To identify chimeric sequences, a reference-based chimera detection step was conducted using the VSEARCH tool against the SILVA gold database ${ }^{23,24}$. Next, the sequence reads were clustered into operational taxonomic units (OTUs) using VSEARCH with a de novo clustering algorithm, with $97 \%$ sequence similarity as the threshold. The representative sequences of the OTUs were finally classified using the SILVA 128 database with UCLUST (parallel_assign_taxonomy_uclust.py script on QIIME version 1.9.1) and default parameters ${ }^{25}$.

\section{Tissue processing and flow cytometric analysis for ILC2s}

Sinonasal tissues were minced using sterile scissors. The tissues were then strained through a $70-\mu \mathrm{m}$ nylon mesh cell strainer into $50-\mathrm{ml}$ polypropylene tubes containing PBS and 1\% fetal bovine serum (FBS; MP Biomedicals, Aurora, OH, USA). Tissue pellets were obtained by centrifugation for $5 \mathrm{~min}$ at $600 \times g$.

For intracellular cytokine staining, a single cell suspension was stimulated with $25 \mathrm{ng} / \mathrm{ml}$ phorbol 12 myristate 13 -acetate and $500 \mathrm{ng} / \mathrm{ml}$ ionomycin (Thermo Fisher Scientific) in the presence of Protein Transport Inhibitor Cocktail (Thermo Fisher Scientific) for $3 \mathrm{~h}$ at $37^{\circ} \mathrm{C}$. The Intracellular Fixation \& Permeabilization Buffer Set (eBioscience, San Diego, CA, USA) was used for cell permeabilization, staining, and subsequent washing. The following antibodies were used at the concentrations recommended by the manufacturers: allophycocyanin (APC)-eFlour780-conjugated anti-CD45; fluorescein isothiocyanate-conjugated lineage markers (anti-CD1a, anti-CD11c, anti-CD14, anti-CD19, anti-CD34, antiCD94, anti-CD123, anti-CD303 (201 A), anti-T-cell receptor (TCR) $\alpha \beta$, anti-TCR $\gamma \delta$, and anti-FceRIa); eFluo450-conjugated anti-CD127; phycoerythrin (PE)Cy7-conjugated anti-161; APC-conjugated anti-CRTH2; and PE-conjugated anti-IL-5 (Thermo Fisher Scientific). The fixable viability dye eFluor 506 (eBioscience) was added to exclude dead cells. Cells were fixed with $200 \mu \mathrm{l}$ of $1 \%$ paraformaldehyde. Data were acquired using a FACSCanto II apparatus (BD Biosciences, Santa Clara, CA, USA) and were analyzed with FlowJo software (Tree Star, Ashland, OR, USA). IL-5-producing ILC2s were identified as lineage ${ }^{-} \mathrm{CD} 45^{+} \mathrm{CD} 127^{+} \mathrm{CD} 161^{+} \mathrm{CRTH} 2^{+} \mathrm{IL}-$ $5^{+}$cells $^{26}$. The prevalence of IL-5-producing ILC2s was calculated as the number of IL- $5^{+}$ILC2s divided by the total number of $\mathrm{CD} 45^{+}$cells.

\section{ELISA for cytokines and chemokines in human tissue homogenates}

Fresh tissue was weighed and homogenized with $1 \mathrm{ml}$ of a mixture containing Protease Inhibitor Cocktail (Roche, Basel, Switzerland), Phosphatase Inhibitor Cocktail (Roche), $150 \mathrm{mM}$ sodium chloride, 1\% Triton X-100, 0.5\% sodium deoxycholate, $0.1 \%$ sodium dodecyl sulfate, $50 \mathrm{mM}$ Tris- $\mathrm{HCl}$, and $2 \mathrm{mM}$ EDTA per $100 \mathrm{mg}$ of tissue. The homogenates were centrifuged at $11,000 \times g$ for $10 \mathrm{~min}$ at $4{ }^{\circ} \mathrm{C}$. The supernatants were collected and stored at $-80^{\circ} \mathrm{C}$ until analysis. IL-5, IL-8, IL-13, interferon-gamma (IFN- $\gamma$ ), $\mathrm{C}-\mathrm{C}$ motif chemokine (CCL) 11, and CCL24 levels were measured using a DuoSet human ELISA kit (R\&D Systems, Minneapolis, MN, USA) according to the manufacturer's instructions.

\section{Statistical analyses}

The demographic and clinical variables were compared using the Kruskal-Wallis test or Chi-square test. Standard ecological diversity indices (e.g., Chaol and Shannon diversity) were assessed using the Kruskal-Wallis test. 
Beta diversity (distance between samples based on differences in OTUs present in each sample) was measured using principal coordinate analysis ( $\mathrm{PCoA}$ ) based on Bray-Curtis dissimilarity. A heat map was used to visualize ordination. Comparison of the relative abundance (RA) of OTUs between groups was performed using the Wilcoxon rank sum test.

Linear discriminant analysis (LDA) effect size (LEfSe) was used to determine genera that best characterized each group (control vs. eosinophilic CRSwNPs vs. noneosinophilic CRSwNPs). LEfSe uses LDA to estimate the effect size of each differentially rich feature ${ }^{27}$. An LDA score $>3$ and $P<0.05$ were considered significant. Correlations were evaluated using Pearson's correlation analysis. Statistical analyses were performed with the $\mathrm{R}$ software package. Statistical significance was evident as $P<0.05$.

\section{Results}

Eosinophilic CRSwNPs patients were older and had higher asthma incidence, polyp grade, Lund-Kennedy endoscopic score, and Lund-Mackay CT score than control and noneosinophilic CRSwNPs patients. Noneosinophilic CRSwNPs patients had a higher prevalence of purulent discharge and saline irrigation in the 6 months before surgery than eosinophilic CRSwNPs patients (Table 1).

Sinus bacterial alpha diversity did not differ significantly among control, eosinophilic CRSwNPs, and noneosinophilic
CRSwNPs subjects (Fig. E1a). At the phylum level, analysis of OTUs demonstrated a rich set of bacterial taxa in both control subjects and patients dominated by characteristic bacterial phyla that included Firmicutes (mainly Staphylococci), Actinobacteria (mainly Corynebacterium, Bifidobacterium, and Propionibacterium species), and Proteobacteria (mainly Moraxella, Pseudomonas, Enterobacter, and Aggregatibacter). Sinus bacteria did not differ at the phylum level between control subjects, eosinophilic CRSwNPs patients, and noneosinophilic CRSwNPs patients (Fig. E1b).

However, sinus bacterial compositions were different at the genus level among control subjects, eosinophilic CRSwNPs patients, and noneosinophilic CRSwNPs patients (Fig. 1a). In eosinophilic CRSwNPs patients, the RAs of Anaerococcus and Tepidimonas were significantly decreased $(P=0.010$ and $P=0.009$, respectively), while that of Lachnoclostridium was significantly increased $(P=0.038)$ when compared with those in controls. The RA of Stenotrophomonas was significantly increased in eosinophilic CRSwNPs patients compared with that in noneosinophilic CRSwNPs patients $(P=0.038)$, the RA of Lachnospiraceae NK4A136 was increased in noneosinophilic CRSwNPs patients compared with that in controls $(P=0.039)$, and the RA of Mesorhizobium was significantly decreased in eosinophilic CRSwNPs patients compared with that in controls $(P=0.012)$ (Fig. 1b). When LEfSe was used to compare bacterial abundance at

Table 1 Preoperative demographic characteristics of subjects.

\begin{tabular}{|c|c|c|c|c|}
\hline Characteristics & Control $(n=6)$ & $\begin{array}{l}\text { Noneosinophilic CRSwNPs } \\
(n=10)\end{array}$ & $\begin{array}{l}\text { Eosinophilic CRSwNPs } \\
(n=21)\end{array}$ & $P$ value \\
\hline Age $(\text { years })^{a}$ & $33.7 \pm 11.4$ & $37.3 \pm 13.9$ & $50.6 \pm 13.5$ & 0.011 \\
\hline Male (\%) & 66.7 & 70.0 & 66.7 & 0.982 \\
\hline Atopy (\%) & 0.0 & 30.0 & 47.6 & 0.091 \\
\hline Asthma (\%) & 0.0 & 0.0 & 38.1 & 0.020 \\
\hline Never smoker (\%) & 83.3 & 70.0 & 66.7 & 0.733 \\
\hline Purulent discharge (\%) & 0 & 80.0 & 66.7 & 0.004 \\
\hline $\begin{array}{l}\text { Systemic antibiotics from } 6 \text { to } 1 \text { months before } \\
\text { surgery (\%) }\end{array}$ & 0.0 & 38.1 & 40.0 & 0.178 \\
\hline Saline irrigation in 6 months before surgery (\%) & 0 & 50.0 & 4.8 & 0.003 \\
\hline Davos polyp classification $^{a}$ & 0 & $3.5 \pm 1.8$ & $4.0 \pm 1.6$ & $<0.001$ \\
\hline Lund-Kennedy endoscopic score ${ }^{a}$ & 0 & $9.3 \pm 2.8$ & $10.1 \pm 2.4$ & $<0.001$ \\
\hline Lund-Mackay CT score ${ }^{a}$ & 0 & $15.6 \pm 5.1$ & $18.0 \pm 3.9$ & $<0.001$ \\
\hline Preoperative SNOT-22 score ${ }^{a}$ & $40.2 \pm 32.5$ & $42.0 \pm 22.7$ & $42.3 \pm 23.2$ & 0.555 \\
\hline Blood eosinophils $(/ \mu l)^{\mathrm{a}}$ & $240.9 \pm 184.7$ & $225.8 \pm 230.1$ & $386.6 \pm 237.6$ & 0.076 \\
\hline Serum total $\lg \mathrm{E}\left(\mathrm{kU} / \mathrm{l}^{\mathrm{a}}\right.$ & $35.4 \pm 48.3$ & $98.4 \pm 65.3$ & $226.6 \pm 192.3$ & 0.016 \\
\hline
\end{tabular}

SNOT sinonasal outcome test.

${ }^{\mathrm{a}}$ Mean \pm standard deviation. 


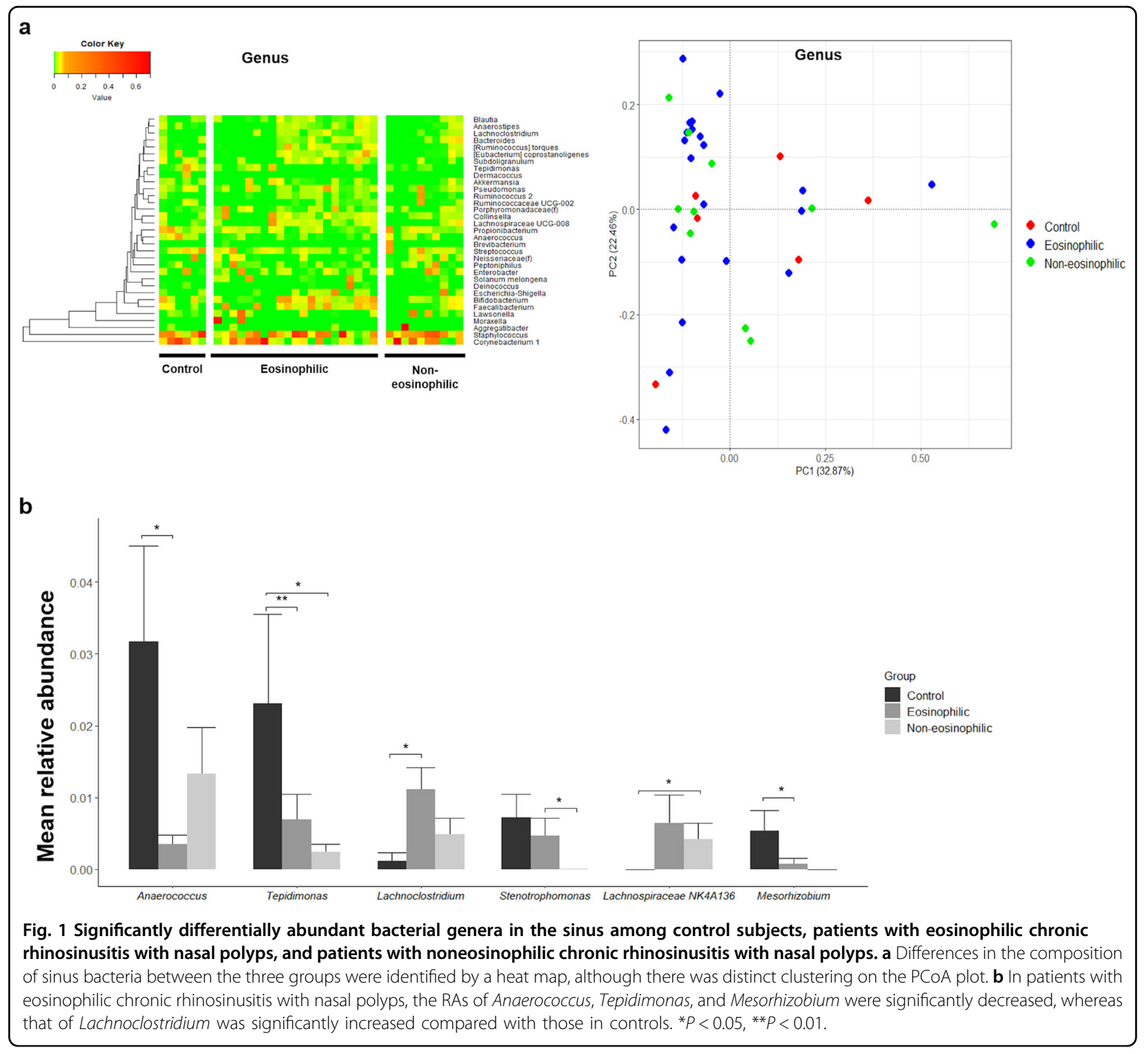

the genus level, Anaerococcus and Tepidimonas were significantly enriched in control subjects (Fig. 2), similar to the results of the comparison between groups.

Correlations between immunological features, such as ILCs, cytokines, and chemokines, and distinct bacteria (Anaerococcus, Tepidimonas, Lachnoclostridium, Stenotrophomonas, and Lachnospiraceae NK4A136) were analyzed. The RA of Lachnoclostridium was significantly positively correlated with IL-5-producing ILC2 populations $(R=0.340, P=0.049$; Fig. E2) without a significant correlation with IL-5 levels $(R=0.188, P=0.266)$. Furthermore, IL-5-producing ILC2 populations correlated with IL-5 $(R=0.351, P=0.042)$ and CCL24 $(R=0.351$, $P=0.042$ ) levels in tissues. The RA of Anaerococcus showed a negative correlation with IL-5-producing ILC2 populations $(R=-0.332, P=0.055)$ and a significant negative correlation with IL-5 levels in tissues $(R=$ $-0.420, P=0.010$; Table 2). There were significant correlations between the levels of other cytokines and chemokines (IL-8, IL-13, IFN- $\gamma$, CCL11, and CCL24) and the RAs of Anaerococcus and Lachnoclostridium. The RAs of Tepidimonas, Stenotrophomonas, and Lachnospiraceae NK4A136 were not correlated with ILCs, cytokines, and chemokines (all $P>0.05$; Table E1).

Patients with suboptimal outcomes exhibited significantly decreased abundances of Corynebacterium at the time of surgery compared with those of patients with optimal outcomes and controls (6.9\% RA vs. $15.3 \%$ RA vs. 19.0\% RA, $P=0.049$ ). The RAs of Anaerococcus and Tepidimonas were also significantly decreased in patients 


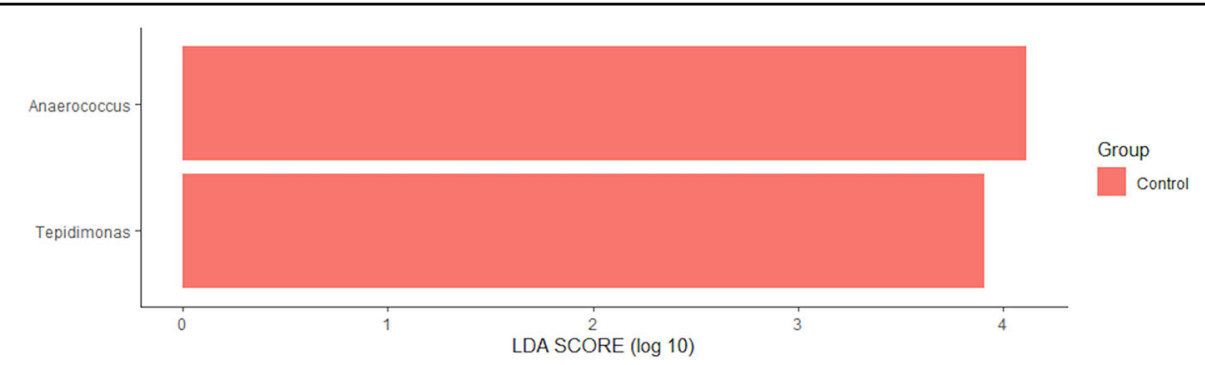

Fig. 2 Linear discriminant analysis (LDA) showing distinct bacterial genera in the sinus. Significantly differentially abundant genera with $P<0.05$ and LDA score $>3$ were observed.

Table 2 Correlation between sinus bacteria and IL5producing group 2 innate lymphoid cells (ILC2s) and IL-5 levels in sinonasal tissue.

\begin{tabular}{|c|c|c|c|c|}
\hline \multirow[t]{2}{*}{ Genera } & \multicolumn{2}{|c|}{$\begin{array}{l}\text { IL5- } \\
\text { producing ILC2 }\end{array}$} & \multicolumn{2}{|l|}{ IL-5 } \\
\hline & $R$ & $P$ & $R$ & $P$ \\
\hline Anaerococcus & -0.332 & 0.055 & -0.420 & 0.010 \\
\hline Tepidimonas & -0.119 & 0.502 & -0.034 & 0.843 \\
\hline Lachnoclostridium & 0.340 & 0.049 & 0.188 & 0.266 \\
\hline Stenotrophomonas & 0.195 & 0.269 & 0.164 & 0.332 \\
\hline Lachnospiraceae & -0.045 & 0.801 & 0.054 & 0.751 \\
\hline
\end{tabular}

with suboptimal outcomes compared with those in patients with optimal outcomes and control subjects (0.4\% RA vs. $0.6 \%$ RA vs. $3.2 \%$ RA, $P=0.033$ and $0.3 \%$ RA vs. $0.8 \%$ RA vs. $2.3 \%$ RA, $P=0.197$, respectively; Fig. 3 ). Among patients with optimal outcomes, the RA of Subdoligranulum was increased in eosinophilic CRSwNPs patients compared with that in noneosinophilic CRSwNPs patients $(0.9 \%$ RA vs. $0.0 \%$ RA, $P=0.026)$. Among patients with suboptimal outcomes, the RA of Klebsiella was increased in eosinophilic CRSwNPs patients compared with that in noneosinophilic CRSwNPs patients ( $0.3 \%$ RA vs. $0.0 \%$ RA, $P=0.045$ ), while the RAs of Gordonia and Ruminococcus gnavus were decreased in eosinophilic CRSwNPs patients compared with those in noneosinophilic CRSwNPs patients (0.0\% RA vs. $0.3 \%$ RA, $P=0.049$ for each).

A moderate-to-strong positive correlation between sinus bacteria and serum EVs was evident for Deinococcus $(R=0.921, \quad P<0.001)$, Sphingomonas $(R=0.599, \quad P=$ $0.009)$, and Lactobacillus $(R=0.423, P=0.009)$. Moreover, there was a strong positive correlation between Deinococcus in sinus bacteria and Enterobacter in serum EVs $(R=0.929, P<0.001)$, between Corynebacterium in sinus bacteria and Neisseriaceae in serum EVs $(R=0.826$, $P<0.001$ ), between Bifidobacterium in sinus bacteria and
Propionibacterium in serum EVs $(R=0.683, P=0.002)$, between Staphylococcus in sinus bacteria and Lactobacillus in serum EVs $(R=0.599, P=0.009)$, and between Anaerococcus in sinus bacteria and Lachnospiraceae. UCG.008 in serum EVs $(R=0.547, P=0.019$; Fig. 4).

\section{Discussion}

Various microbes, including bacteria, viruses, and fungi, thrive in the human body in a symbiotic relationship ${ }^{28}$. Many microbiota-related studies have been performed with the knowledge that the microbiota strongly influences human health and diseases ${ }^{29}$. These studies have been performed not only for the gut but also for the respiratory tract, which is one of the entry routes of bacteria into the body. Bacteria have been implicated in the pathogenesis of CRS, and staphylococcal superantigen, immune barrier, and biofilm hypotheses have been proposed ${ }^{30-32}$. However, the exact roles of bacteria in CRS remain controversial.

In the present study, eosinophilic CRSwNPs patients showed significantly lower RAs of Anaerococcus and Tepidimonas and a higher RA of Lachnoclostridium at the genus level than controls and noneosinophilic CRSwNPs patients. LEfSe also showed Anaerococcus and Tepidimonas enrichment in controls. The clinical and demographic variables were different among controls, eosinophilic CRSwNPs patients, and noneosinophilic CRSwNPs patients. These variables might affect bacterial composition. Eosinophilic CRSwNPs patients had higher asthma incidence and clinical severity scores but lower purulence incidence and saline irrigation than noneosinophilic CRSwNPs patients. These findings indicate that asthma, clinical severity, and purulence may be associated with significant alterations in bacterial composition, similar to the findings of a previous study ${ }^{17}$, although a direct relationship was not analyzed. The three groups showed similar rates of smoking, systemic antibiotic administration before surgery, and saline irrigation. Given that these factors were not associated with distinct alterations in sinonasal bacteria ${ }^{33-35}$, their effects on the microbiome might not be significant. 


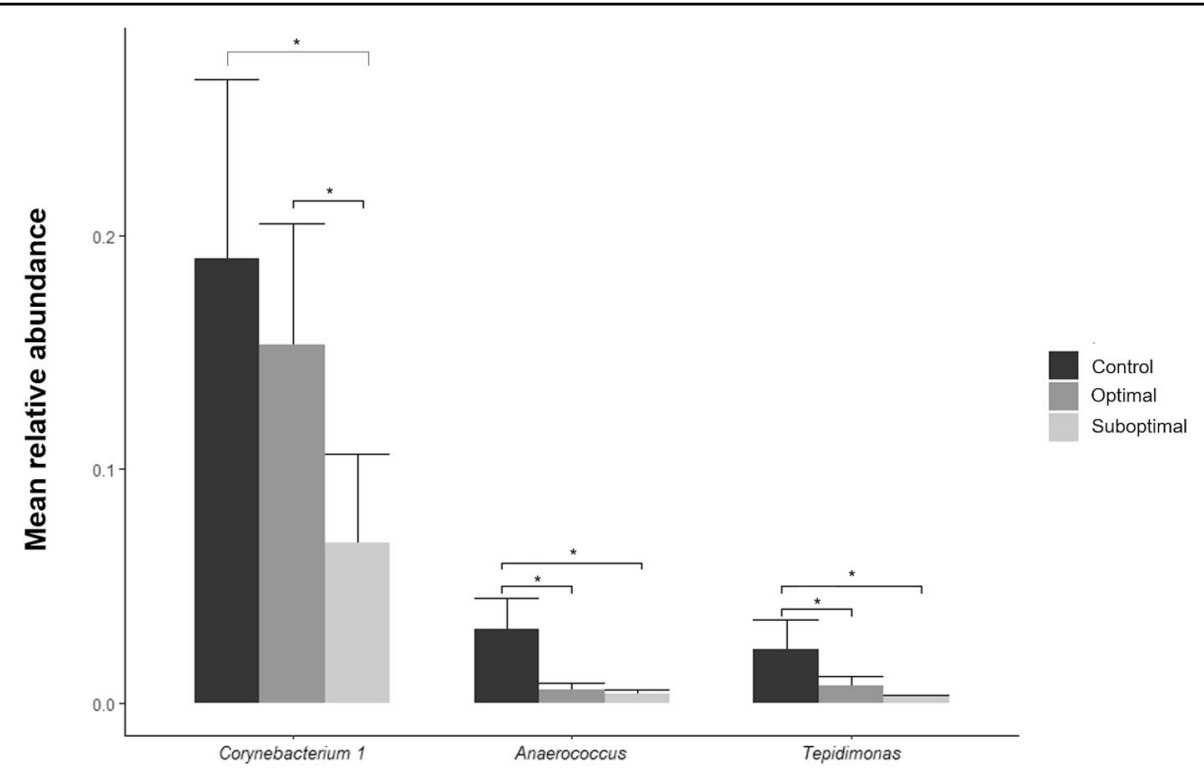

Fig. 3 Significantly differentially abundant bacterial genera in the sinus according to the surgical outcome. Corynebacterium, Anaerococcus, and Tepidimonas were significantly less abundant in patients with suboptimal outcomes than in those with optimal outcomes and control subjects. *P $<0.05$.

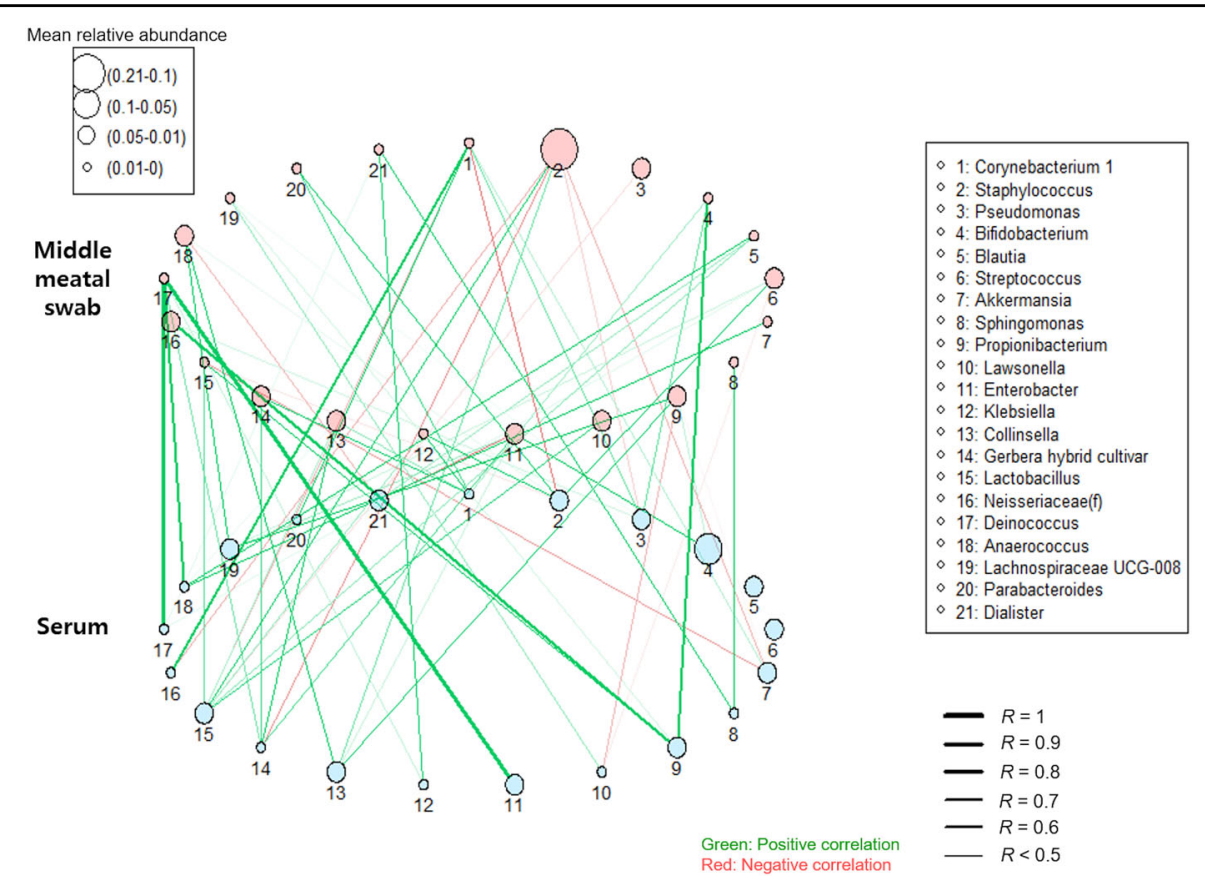

Fig. 4 Correlation between sinus bacteria and serum extracellular vesicles. Deinococcus, Sphingomonas, and Lactobacillus among the sinus bacteria were positively correlated with serum extracellular vesicles.

In one study, when a CRS patient cluster was divided into distinct subgroups, each subgroup was associated with a distinct metabolism and host immune response ${ }^{7}$. The immunologic features associated with the nature of the sinus microbiota can partially explain CRS heterogeneity and can be used as a basis for establishing a customized treatment strategy. In our study, the IL-5producing ILC2 population showed a significant positive correlation with the RA of Lachnoclostridium but a negative correlation with the RA of Anaerococcus. The 
observation that IL-5-producing ILC2 populations correlated with the mediators of eosinophilic inflammation, such as IL-5 and CCL24 levels in tissues, might explain the RA of Lachnoclostridium and the reduction in Anaerococcus abundance in eosinophilic CRSwNPs patients. Increased Lachnoclostridium abundance with type 2 responses and gut dysbiosis was suggested as a mechanism of food allergy induction during consumption of a high-fat $\operatorname{diet}^{36}$. Knowledge about the association between Anaerococcus and the immune response is limited. Given that $A$. prevotii is frequently identified from vaginal discharges and ovarian, peritoneal, sacral, or lung abscesses $^{37}$, Anaerococcus may be related to the type 1 response.

$\mathrm{CD} 4^{+} \mathrm{T}$ helper (Th) cells play an important role in coordinating adaptive immune responses. Endotypes of CRS are differentiated mainly on the basis of Th cells other than ILCs, which produce cytokines and chemokines, leading to the accumulation of eosinophils and neutrophils ${ }^{38}$. The bacteria are associated with the endotypes of CRS in relation to cytokines produced by Th cells. Gram-negative bacteria were isolated more frequently in IL-5/IL-17/IFN- $\gamma$-negative NPs patients, whereas gram-positive bacteria were more associated with IL-5-positive NPs patients ${ }^{39}$. Moreover, depletion of commensal bacteria using oral broad-spectrum antibiotics increases serum IgE levels and exacerbates basophilmediated Th2 cell responses and allergic inflammation in the airway ${ }^{40}$.

Bacterial diversity and composition were previously suggested as predictors of surgical outcome. In 27 patients with CRS, more diverse bacterial communities and enriched Actinobacteria at the phylum level and Corynebacterium at the genus level showed optimal outcomes than suboptimal outcomes ${ }^{17}$. Similarly, in our study, patients with optimal outcomes were more enriched for the genera Corynebacterium, Anaerococcus, and Tepidimonas at the time of surgery than those with suboptimal outcomes and less enriched than controls.

EVs circulate through the blood and directly and/or indirectly communicate with tissues and organs ${ }^{10}$. Blood bacterial EVs were suggested to be useful for metagenome analysis of the bodily microbiota in preterm birth and a mouse model of Alzheimer's disease ${ }^{41,42}$. In this study, Deinococcus, Sphingomonas, and Lactobacillus among the sinus bacteria were positively correlated with serum EVs. Individual bacteria in the middle meatus and serum were also correlated. These correlations suggested that sinus bacteria might communicate with blood bacterial EVs. Although direct causality between sinus bacteria and blood bacterial EVs and the mechanism of the latter's formation have not been proven, Sphingomonas may be potentially meaningful in terms of sinus bacteria and blood bacteria communication. The Sphingomonadaceae family was found to be abundant in the urine-derived EVs of children with asthma and chronic rhinitis compared with that in EVs of controls ${ }^{43}$. Sphingomonas species in the airway were associated with increased airway hyperresponsiveness to methacholine, positively correlated with eosinophil cationic protein levels, and strongly and positively correlated with serum IgE levels and blood and sputum eosinophil counts in patients with asthma ${ }^{44-46}$. This association may be explained by glycosphingolipid, a cell membrane component of Sphingomonas that activates natural killer $\mathrm{T}$ cells producing IL-4 and IL-13 ${ }^{47-49}$. Further research is needed on the association of Sphingomonas between the sinus and serum in relation to type 2 immune responses and eosinophilic CRSwNPs. Another potentially interesting bacterium is Lactobacillus; its RA in the sinus is less than that of other bacteria, but its sinus RA is positively correlated with its RA in serum EVs. Considering that Lactobacillus has been suggested as a potential protective species against allergens and virus infection $^{50,51}$, deficiency in Lactobacillus in the sinus and blood may be associated with the pathophysiology of CRS.

The current study included a small number of subjects. However, the observed relationship between immunological characteristics in terms of ILCs and bacteria in the sinonasal cavity in CRSwNPs patients is a novel finding. Because there was large variation in the bacterial composition of the sinonasal cavity between individuals, similar to that in a previous study ${ }^{7}$, clustering based on the clinical characteristics was not distinct. Bacterial metabolites were not investigated in this study. A previous study demonstrated that the Streptococcaceae dominant group, Pseudomonadaceae dominant group, Corynebacteriaceae dominant group, and Staphylococcaceae dominant group encoded enriched gene pathways, including ansamycin biosynthesis, tryptophan metabolism, the two-component response system virulence pathway, and the peroxisome proliferator-activated receptor gamma signaling pathway, respectively. These metabolites may be involved in the immune responses of nasal polyp tissues ${ }^{7}$. Therefore, the effect of the metabolites of Lachnoclostridium and Anaerococcus on ILC2s in NP tissues should be further investigated.

Finally, this study showed that the sinus microbiota, particularly Anaerococcus and Lachnoclostridium in CRSwNPs patients, was associated with eosinophilic inflammation and IL-5-producing ILC2s. The abundances of the genera Corynebacterium, Anaerococcus, and Tepidimonas were related to surgical outcome. Furthermore, some sinus bacteria were correlated with blood bacterial EVs. Therefore, understanding the sinus microbiota of individuals could be the basis for effective treatment of CRS and for predicting prognosis. 


\section{Acknowledgements}

This study was supported by a grant (2018-769) from the Asan Institute for Life Sciences, Asan Medical Center, Seoul, Republic of Korea and by the Basic Science Research Program through the National Research Foundation of Korea (NRF), funded by the Ministry of Education (2017R1D1A1B03031452). These funders played no role in the study design, data collection and analysis, decision to publish, or manuscript preparation.

\section{Conflict of interest}

The authors declare that they have no conflict of interest.

\section{Publisher's note}

Springer Nature remains neutral with regard to jurisdictional claims in published maps and institutional affiliations.

Supplementary information accompanies this paper at https://doi.org/ 10.1038/s12276-020-0458-1.

Received: 13 January 2020 Revised: 10 April 2020 Accepted: 15 April 2020. Published online: 29 June 2020

\section{References}

1. Sivasubramaniam, R. \& Douglas, R. The microbiome and chronic rhinosinusitis. World J. Otorhinolaryngol. Head. Neck Surg. 4, 216-221 (2018).

2. Fokkens, W. J. et al. European position paper on rhinosinusitis and nasal polyps 2012. Rhinol. Suppl. 23, 1-298 (2012).

3. Lam, K., Schleimer, R. \& Kern, R. C. The etiology and pathogenesis of chronic rhinosinusitis: a review of current hypotheses. Curr. Allergy Asthma Rep. 15, 41 (2015).

4. Abreu, N. A. et al. Sinus microbiome diversity depletion and Corynebacterium tuberculostearicum enrichment mediates rhinosinusitis. Sci. Transl. Med. 4, 151ra124 (2012)

5. Biswas, K., Hoggard, M., Jain, R., Taylor, M. W. \& Douglas, R. G. The nasal microbiota in health and disease: variation within and between subjects. Front. Microbiol. 9, 134 (2015).

6. Tomassen, P. et al. Inflammatory endotypes of chronic rhinosinusitis based on cluster analysis of biomarkers. J. Allergy Clin. Immunol. 137, 1449-1456.e4 (2016).

7. Cope, E. K., Goldberg, A. N., Pletcher, S. D. \& Lynch, S. V. Compositionally and functionally distinct sinus microbiota in chronic rhinosinusitis patients have immunological and clinically divergent consequences. Microbiome 5, 53 (2017).

8. Tojima, l. et al. Group 2 innate lymphoid cells are increased in nasal polyps in patients with eosinophilic chronic rhinosinusitis. Clin. Immunol. 170, 1-8 (2016).

9. Sonnenberg, G. F. \& Artis, D. Innate lymphoid cell interactions with microbiota: implications for intestinal health and disease. Immunity $\mathbf{3 7}$ 601-610 (2012).

10. Théry, C., Ostrowski, M. \& Segura, E. Membrane vesicles as conveyors of immune responses. Nat. Rev. Immunol. 9, 581-593 (2009).

11. Bhatnagar, S., Shinagawa, K., Castellino, F. J. \& Schorey, J. S. Exosomes released from macrophages infected with intracellular pathogens stimulate a proinflammatory response in vitro and in vivo. Blood 110, 3234-3244 (2007).

12. Pyun, B. Y. Extracellular vesicle: an unknown environmental factor for causing airway disease. Allergy Asthma Immunol. Res. 8, 179-180 (2016).

13. Hong, S. W. et al. Extracellular vesicles derived from Staphylococcus aureus induce atopic dermatitis-like skin inflammation. Allergy $\mathbf{6 6}$, 351-359 (2011)

14. Choi, E. B. et al. Decreased diversity of nasal microbiota and their secreted extracellular vesicles in patients with chronic rhinosinusitis based on a metagenomic analysis. Allergy 69, 517-526 (2014).

15. Cao, P. P. et al. Distinct immunopathologic characteristics of various types of chronic rhinosinusitis in adult Chinese. J. Allergy Clin. Immunol. 124, 478-484, e1-2 (2009).

16. Lee, $M$. et al. Sirtuin 1 attenuates nasal polypogenesis by suppressing epithelial-to-mesenchymal transition. J. Allergy Clin. Immunol. 137, 87-98.e7 (2016).
17. Ramakrishnan, V. R. et al. Sinus microbiota varies among chronic rhinosinusitis phenotypes and predicts surgical outcome. J. Allergy Clin. Immunol. 136, 334-342.e1 (2015).

18. Yang, J. et al. Consumption of a Leuconostoc holzapfelii-enriched synbiotic beverage alters the composition of the microbiota and microbial extracellular vesicles. Exp. Mol. Med. 51, 87 (2019).

19. Yang, J. et al. Development of a colorectal cancer diagnostic model and dietary risk assessment through gut microbiome analysis. Exp. Mol. Med. 51, 117 (2019).

20. Martin, M. Cutadapt removes adapter sequences from high-throughput sequencing reads. EMBnet J. 17, 10-12 (2011).

21. Kwon, S., Lee, B. \& Yoon, S. CASPER: context-aware scheme for paired-end reads from high-throughput amplicon sequencing. BMC Bioinforma. 9(15 Suppl), S10 (2014)

22. Bokulich, N. A. et al. Quality-filtering vastly improves diversity estimates from Illumina amplicon sequencing. Nat. methods 10, 57 (2013).

23. Rognes, T., Flouri, T., Nichols, B., Quince, C. \& Mahé, F. VSEARCH: a versatile open source tool for metagenomics. PeerJ 4, e2584 (2016).

24. Quast, C. et al. The SILVA ribosomal RNA gene database project: improved data processing and web-based tools. Nucleic acids Res. 41 D590-D596 (2012)

25. Caporaso, J. G. et al. QIIME allows analysis of high-throughput community sequencing data. Nat. methods 7, 335 (2010).

26. Hazenberg, M. D. \& Spits, H. Human innate lymphoid cells. Blood 124, 700-709 (2014).

27. Segata, N. et al. Metagenomic biomarker discovery and explanation. Genome Biol. 12, R60 (2011).

28. Gill, S. R. et al. Metagenomic analysis of the human distal gut microbiome. Science 312, 1355-1359 (2006).

29. NIH HMP Working Group. The NIH Human Microbiome Project. Genome Res. 19, 2317-2323 (2009).

30. Bachert, C., Zhang, N., Patou, J., van, Zele, T. \& Gevaert, P. Role of staphylococcal superantigens in upper airway disease. Curr. Opin. Allergy Clin. Immunol. 8 34-38 (2008)

31. Kern, R. C. et al. Perspectives on the etiology of chronic rhinosinusitis: an immune barrier hypothesis. Am. J. Rhinol. 22, 549-559 (2008).

32. Foreman, A. et al. Adaptive immune responses in Staphylococcus aureus biofilm-associated chronic rhinosinusitis. Allergy 66, 1449-1456 (2011).

33. Ramakrishnan, V. R. \& Frank, D. N. Impact of cigarette smoking on the middle meatus microbiome in health and chronic rhinosinusitis. Int. Forum Allergy Rhinol. 5, 981-989 (2015).

34. Merkley, M. A. et al. The effect of antibiotics on the microbiome in acute exacerbations of chronic rhinosinusitis. Int. Forum Allergy Rhinol. 5, 884-893 (2015).

35. Liu, C. M. et al. Impact of saline irrigation and topical corticosteroids on the postsurgical sinonasal microbiota. Int. Forum Allergy Rhinol. 5, 185-190 (2015).

36. Hussain, M. et al. High dietary fat intake induces a microbiota signature that promotes food allergy. J. Allergy Clin. Immunol. 144, 157-170.e8 (2019).

37. Labutti, K. et al. Complete genome sequence of Anaerococcus prevotii type strain. Stand Genom. Sci. 1, 159-165 (2009).

38. Bachert, C. \& Akdis, C. A. Phenotypes and emerging endotypes of chronic rhinosinusitis. J. Allergy Clin. Immunol. Pract. 4, 621-628 (2016).

39. Ba., L. et al. The association between bacterial colonization and inflammatory pattern in chinese chronic rhinosinusitis patients with nasal polyps. Allergy $\mathbf{6 6}$ 1296-1303 (2011).

40. Hill, A. D. et al. Commensal bacteria-derived signals regulate basophil hematopoiesis and allergic inflammation. Nat. Med. 18, 538-546 (2012).

41. You, Y. A., Yoo, J. Y., Kwon, E. J. \& Kim, Y. J. Blood microbial communities during pregnancy are associated with preterm birth. Front. Microbiol. 10 1122 (2019).

42. Park, J. Y. et al. Metagenome analysis of bodily microbiota in a mouse mode of Alzheimer disease using bacteria-derived membrane vesicles in blood. Exp. Neurobiol. 26, 369-379 (2017).

43. Sarma, M. et al. Urine bacteria-derived extracellular vesicles and allergic airway diseases in children. Int. Arch. Allergy Immunol. 178, 150-158 (2019).

44. Huang, Y. J. et al. Airway microbiota and bronchial hyperresponsiveness in patients with suboptimally controlled asthma. J. Allergy Clin. Immunol. 127, 372-381.e1-3 (2011).

45. Sverrild, A. et al. Eosinophilic airway inflammation in asthmatic patients is associated with an altered airway microbiome. J. Allergy Clin. Immunol. 140 407-417.e11 (2017). 
46. Durack, J. et al. Features of the bronchial bacterial microbiome associated with atopy, asthma, and responsiveness to inhaled corticosteroid treatment. J. Allergy Clin. Immunol. 140, 63-75 (2017).

47. Akbari, O. et al. Essential role of NKT cells producing IL-4 and IL-13 in the development of allergen-induced airway hyperreactivity. Nat. Med. 9, 582-588 (2003).

48. Kinjo, Y. et al. Recognition of bacterial glycosphingolipids by natural killer T cells. Nature 434, 520-525 (2005)
49. Zajonc, D. M, Girardi. E. Recognition of microbial glycolipids by natural killer T cells. Front. Immunol. 6, 400 (2015)

50. Abreu, N. A. et al. Sinus microbiome diversity depletion and Corynebacterium Tuberculostearicum enrichment mediates rhinosinusitis. Sci. Transl. Med. 4 151-124 (2012).

51. Fujimura, K. E. et al. House dust exposure mediates gut microbiome Lactobacillus enrichment and airway immune defense against allergens and virus infection. Proc. Natl Acad. Sci. USA. 111, 805-810 (2014). 